\title{
An Optimization Problem Related to Minkowski's Successive Minima
}

\author{
Romanos Malikiosis
}

Received: 13 November 2008 / Revised: 24 February 2009 / Accepted: 25 February 2009 /

Published online: 21 March 2009

(C) The Author(s) 2009. This article is published with open access at Springerlink.com

\begin{abstract}
The purpose of this paper is to establish an inequality connecting the lattice point enumerator of a 0 -symmetric convex body with its successive minima. To this end, we introduce an optimization problem whose solution refines former methods, thus producing a better upper bound. In particular, we show that an analogue of Minkowski's second theorem on successive minima with the volume replaced by lattice point enumerator is true up to an exponential factor, whose base is approximately 1.64 .
\end{abstract}

Keywords Successive minima $\cdot$ Lattice points

\section{Introduction}

Let $\mathcal{K}_{0}^{d}$ denote the set of all compact, $d$-dimensional, 0 -symmetric convex bodies for which $0 \in \operatorname{int}(K)$, and let $K \in \mathcal{K}_{0}^{d}$ be arbitrary. We denote by $G(K, \Lambda)$ the lattice point enumerator in $K$ with respect to the lattice $\Lambda$, i.e., $\#(K \cap \Lambda)$, and let $\operatorname{vol}(K)$ denote the usual $d$-dimensional Lebesgue measure of $K$. The $i$ th successive minimum of $K$ with respect to the lattice $\Lambda$, denoted by $\lambda_{i}=\lambda_{i}(K, \Lambda)(1 \leq i \leq d)$, is defined as follows:

$$
\lambda_{i}=\inf \{\lambda>0 \mid \lambda K \cap \Lambda \text { contains at least } i \text { linearly independent points }\} .
$$

Obviously,

$$
0<\lambda_{1} \leq \lambda_{2} \leq \cdots \leq \lambda_{d}<+\infty .
$$

Finally, $\operatorname{det}(\Lambda)$ will denote the determinant of a lattice, i.e., the volume of a fundamental parallelotope of $\Lambda$.

R. Malikiosis $(\bowtie)$

Department of Mathematics, UCLA, Box 951555, Los Angeles, CA 90095-1555, USA

e-mail: romanos@math.ucla.edu 
In 1896 (see [2]), Minkowski stated and proved his famous two theorems relating the volume of $K \in \mathcal{K}_{0}^{d}$ with its successive minima. The first theorem is:

Theorem 1.1 Let $K \in \mathcal{K}_{0}^{d}$. Then the following inequality holds:

$$
\operatorname{vol}(K) \leq \operatorname{det}(\Lambda)\left(\frac{2}{\lambda_{1}}\right)^{d}
$$

Minkowski himself used the above to prove a lower bound on the discriminant of a number field. In particular, he deduced that there is no nontrivial algebraic extension of $\mathbb{Q}$ that is unramified at all primes. This is a key ingredient in many deep theorems in number theory. It should be noted that Theorem 1.1 is more widely known in the following equivalent form: If $K \in \mathcal{K}_{0}^{d}$ satisfies $\operatorname{vol}(K) \geq 2^{d} \operatorname{det}(\Lambda)$, then it contains a nontrivial lattice point.

Minkowski's second theorem on successive minima is a stronger result:

Theorem 1.2 Let $K \in \mathcal{K}_{0}^{d}$. Then the following inequality holds:

$$
\operatorname{vol}(K) \leq \operatorname{det}(\Lambda) \prod_{i=1}^{d} \frac{2}{\lambda_{i}}
$$

Besides algebraic number theory, Theorem 1.2 has a wide number of applications in various areas of mathematics, as in Diophantine approximation or adelic geometry of numbers, to name a few, and is considered a very deep result in the geometry of numbers [2].

In 1993, Betke, Henk, and Wills [1] stated analogues of Minkowski's theorems for the lattice point enumerator, instead of the volume. Their first theorem is the following:

Theorem 1.3 Let $K \in \mathcal{K}_{0}^{d}$. Then the following inequality holds:

$$
G(K, \Lambda) \leq\left[\frac{2}{\lambda_{1}}+1\right]^{d}
$$

Here, as usual, $[x]$ denotes the integer part of $x$. An analogue to the second theorem was proven only for the planar case, $d=2$, being trivial for $d=1$ :

Conjecture 1.1 Let $K \in \mathcal{K}_{0}^{d}$. Then the following inequality holds:

$$
G(K, \Lambda) \leq \prod_{i=1}^{d}\left[\frac{2}{\lambda_{i}}+1\right]
$$

It should be noted that the conjecture above, if true, would imply Minkowski's second theorem on successive minima, using a simple argument involving the definition of the Riemann integral [1]. Betke, Henk, and Wills proved that Conjecture 1.1 
holds roughly up to a factor of $d$ !. Later, Henk [3] improved this inequality to

$$
G(K, \Lambda) \leq 2^{d-1} \prod_{i=1}^{d}\left[\frac{2}{\lambda_{i}}+1\right] .
$$

Examining Henk's proof leads us to an optimization problem, in particular, finding a better upper bound for the constant $C_{d}$ defined below:

Definition 1.1 Let $C_{d}$ denote the least positive constant such that, for any sequence of $d$ integers $x_{1} \leq x_{2} \leq \cdots \leq x_{d}$, there exists a sequence of integers $y_{1}, y_{2}, \ldots, y_{d}$ satisfying:

(a) $x_{i} \leq y_{i}$ for all $i, 1 \leq i \leq d$.

(b) $y_{i}$ divides $y_{i+1}$ for all $i, 1 \leq i \leq d-1$.

(c) $\frac{y_{1} y_{2} \cdots y_{d}}{x_{1} x_{2} \cdots x_{d}} \leq C_{d}$.

In the course of proving Theorem 1.4, Henk essentially proved that $C_{d} \leq 2^{d-1}$. Here, we shall prove a better upper bound, as well as a lower bound:

Proposition 1.1 $2^{(d-1) / 2} \leq C_{d} \leq(4 / e) \cdot 3^{(d-1) / 2}$, and the lower bound is tight.

Using the method given in the proof of Proposition 1.1, we were able to improve Theorem 1.4:

Theorem 1.4 Let $K \in \mathcal{K}_{0}^{d}$. Then the following inequality holds:

$$
G(K, \Lambda) \leq \frac{4}{e}\left(\sqrt[3]{\frac{40}{9}}\right)^{d-1} \prod_{i=1}^{d}\left[\frac{2}{\lambda_{i}}+1\right]
$$

We should note that $4 / e \approx 1.47152$ and $\sqrt[3]{40 / 9} \approx 1.64414$. In Sect. 4 , we will prove better inequalities than those described by Theorem 1.4 for the cases $d=3$ and $d=4$ :

Proposition 1.2 Let $K \in \mathcal{K}_{0}^{d}$. If $d=3$, we have

$$
G(K, \Lambda) \leq 2 \prod_{i=1}^{3}\left[\frac{2}{\lambda_{i}}+1\right]
$$

and, if $d=4$,

$$
G(K, \Lambda) \leq \frac{7203}{2375} \prod_{i=1}^{4}\left[\frac{2}{\lambda_{i}}+1\right]
$$

$(7203 / 2375 \approx 3.03284)$. 
The corresponding inequalities derived from Theorem 1.4 for $d=3$ and $d=4$ give

$$
\frac{4}{e}\left(\frac{40}{9}\right)^{2 / 3} \approx 3.97781
$$

and

$$
\frac{4}{e} \cdot \frac{40}{9} \approx 6.54008
$$

\section{Henk's Proof}

Let us review first the proof of inequality (1); we will simply expand the technique used in the proof in order to obtain a better inequality. The following lemma is needed:

Lemma 2.1 Let $K \in \mathcal{K}_{0}^{d}$, and let $\tilde{\Lambda} \subset \Lambda$ be two lattices in $\mathbb{R}^{d}$. Then

$$
G(K, \Lambda) \leq \frac{\operatorname{det} \tilde{\Lambda}}{\operatorname{det} \Lambda} G(2 K, \tilde{\Lambda}) .
$$

For a proof, see [3].

Proposition 2.1 Let $0<x_{1} \leq x_{2} \leq \cdots \leq x_{d}$ be $d$ integer numbers. Then there are integers $y_{1}, y_{2}, \ldots, y_{d}$ satisfying $x_{i} \leq y_{i}, y_{i} \mid y_{i+1}$ for all $i$ and

$$
\frac{y_{1} y_{2} \cdots y_{d}}{x_{1} x_{2} \cdots x_{d}}<2^{d-1}
$$

Proof It suffices to put $y_{1}=x_{1}$ and inductively construct $x_{i} \leq y_{i}<2 x_{i}$ such that $y_{i} \mid y_{i+1}$. Such a construction is possible; assuming that we have constructed $y_{1}, \ldots, y_{k}$ satisfying the above requirements, we will construct $y_{k+1}$. If $x_{k+1} \leq y_{k}$, we simply set $y_{k+1}=y_{k}$. Obviously, $x_{k+1} \leq y_{k+1}<2 x_{k} \leq 2 x_{k+1}$. Otherwise, we consider the euclidean division of $x_{k+1}$ by $y_{k}$, say $x_{k+1}=m \cdot y_{k}+r$, where $0 \leq r<y_{k}$. Then, we set $y_{k+1}=(m+1) y_{k}$, which again satisfies the desired requirements.

Proof of inequality (1) We need the following simple fact; if $a^{1}, \ldots, a^{d}$ are $d$ linearly independent lattice vectors of $\Lambda$, then there is a basis of $\Lambda$, say $e^{1}, \ldots, e^{d}$ such that for all $i$ with $1 \leq i \leq d$, we have

$$
\operatorname{lin}\left(a^{1}, \ldots, a^{i}\right) \subset \operatorname{lin}\left(e^{1}, \ldots, e^{i}\right),
$$

where "lin" denotes the linear hull of the mentioned vectors, i.e., the set of all linear combinations with integer coefficients. Furthermore, if the $a^{i}$,s are such that $a^{i} \in$ $\lambda_{i} K$, then

$$
\operatorname{int}\left(\lambda_{i} K\right) \cap \Lambda \subset \operatorname{lin}\left(0, e^{1}, \ldots, e^{i-1}\right) \cap \Lambda,
$$


which follows from the definition of the successive minima.

We set $q_{i}=\left[2 \lambda_{i}^{-1}+1\right]$. We want to find $d$ integer numbers $n_{i}, 1 \leq i \leq d$, such that $q_{i} \leq n_{i}$ and $n_{i+1} \mid n_{i}$ for $1 \leq i \leq d-1$ and

$$
\frac{n_{1} n_{2} \cdots n_{d}}{q_{1} q_{2} \cdots q_{d}}<2^{d-1}
$$

This is possible by Proposition 2.1. Next, we consider the lattice $\tilde{\Lambda} \subset \Lambda$ which is generated by the vectors $n_{1} e^{1}, \ldots, n_{d} e^{d}$. By the above lemma we have

$$
G(K, \Lambda) \leq G(2 K, \tilde{\Lambda}) \prod_{i=1}^{d} n_{i}<G(2 K, \tilde{\Lambda}) \cdot 2^{d-1} \prod_{i=1}^{d}\left[2 \lambda_{i}^{-1}+1\right] .
$$

Thus, it suffices to prove that $G(2 K, \widetilde{\Lambda})=1$. Assuming otherwise, let $g$ be a nonzero vector that is an element of $2 K \cap \widetilde{\Lambda}$, and let $k$ be the largest index of a nonzero coordinate of $g$. Then, for some $z_{i} \in \mathbb{Z}, 1 \leq i \leq k$, we have

$$
g=z_{1}\left(n_{1} e^{1}\right)+\cdots+z_{k}\left(n_{k} e^{k}\right) \in 2 K .
$$

Since $n_{k}$ divides $n_{1}, \ldots, n_{k-1}$ and $2 / n_{k}<\lambda_{k}$, we obtain

$$
\frac{1}{n_{k}} g \in\left(\frac{2}{n_{k}} K\right) \cap \Lambda \subset \operatorname{int}\left(\lambda_{k} K\right) \cap \Lambda \subset \operatorname{lin}\left(0, e^{1}, \ldots, e^{k-1}\right) \cap \Lambda,
$$

which is a contradiction. Hence, $2 K \cap \widetilde{\Lambda}=\{0\}$ and $G(2 K, \widetilde{\Lambda})=1$, as desired.

\section{An Optimization Problem}

We observe that there is an undesired factor of magnitude $2^{d-1}$, which is obtained from Proposition 2.1. Can we improve this factor? We are naturally led to Definition 1.1, and we will attempt to give a better upper bound.

In order to obtain an estimate on $C_{d}$, we drop the hypothesis on integrality of the $x_{i}$ 's and $y_{i}$ 's; in this setting, $y_{i} \mid y_{i+1}$ means that $y_{i+1} / y_{i} \in \mathbb{Z}$. We call the corresponding constant by $c_{d}$ :

Definition 3.1 Let $c_{d}$ denote the least positive constant such that, for any sequence of $d$ positive real numbers $x_{1} \leq x_{2} \leq \cdots \leq x_{d}$, there exists a sequence of real numbers $y_{1}, y_{2}, \ldots, y_{d}$ satisfying:

(a) $x_{i} \leq y_{i}$ for all $i, 1 \leq i \leq d$.

(b) $y_{i+1} / y_{i} \in \mathbb{Z}$ for all $i, 1 \leq i \leq d-1$.

(c) $\frac{y_{1} y_{2} \cdots y_{d}}{x_{1} x_{2} \cdots x_{d}} \leq c_{d}$.

We will prove later that $c_{d} \leq C_{d}$. The following nice lemma was proven by Rogers [4]. We provide a proof here for convenience (see also [2], p. 190):

Lemma $3.1 c_{d}=2^{(d-1) / 2}$. 
Proof For each $i, 1 \leq i \leq d$, we construct the sequence $y_{1}^{i}, \ldots, y_{d}^{i}$ that satisfies

$$
y_{i}^{i}=x_{i}, \quad y_{j}^{i}=2^{a_{i j}} x_{i} \quad \text { for } j \neq i, \quad \text { where } a_{i j}=-\left[\log _{2} \frac{x_{i}}{x_{j}}\right] .
$$

In other words, $a_{i j}$ is the unique integer satisfying

$$
x_{j} \leq 2^{a_{i j}} x_{i}<2 x_{j} .
$$

Therefore,

$$
\log _{2} \frac{y_{j}^{i}}{x_{j}}=\left\{\log _{2} x_{i}-\log _{2} x_{j}\right\}
$$

for all $j$, so

$$
\log _{2} \frac{y_{1}^{i} \cdots y_{d}^{i}}{x_{1} \cdots x_{d}}=\sum_{j=1}^{d}\left\{\log _{2} x_{i}-\log _{2} x_{j}\right\}
$$

Summing over all $i$, we obtain

$$
\sum_{i=1}^{d} \log _{2} \frac{y_{1}^{i} \cdots y_{d}^{i}}{x_{1} \cdots x_{d}}=\sum_{i, j=1}^{d}\left\{\log _{2} x_{i}-\log _{2} x_{j}\right\} .
$$

For any pair $(i, j)$ with $i \neq j,\left\{\log _{2} x_{i}-\log _{2} x_{j}\right\}+\left\{\log _{2} x_{j}-\log _{2} x_{i}\right\} \leq 1$ (for $i=j$, it vanishes). Since there are $d(d-1) / 2$ such pairs, we get

$$
\sum_{i=1}^{d} \log _{2} \frac{y_{1}^{i} \cdots y_{d}^{i}}{x_{1} \cdots x_{d}} \leq \frac{d(d-1)}{2}
$$

Hence, there is an index $i$ such that

$$
\log _{2} \frac{y_{1}^{i} \cdots y_{d}^{i}}{x_{1} \cdots x_{d}} \leq \frac{d-1}{2}
$$

and hence

$$
\frac{y_{1}^{i} \cdots y_{d}^{i}}{x_{1} \cdots x_{d}} \leq 2^{\frac{d-1}{2}} .
$$

Since the increasing sequence $x_{1}, \ldots, x_{d}$ is arbitrary, we have $c_{d} \leq 2^{(d-1) / 2}$. We will show, by an example, that $c_{d}=2^{(d-1) / 2}$; let $x_{i}=2^{(i-1) / d}$. Let $y_{1}, \ldots, y_{d}$ be an increasing sequence satisfying $x_{i} \leq y_{i}$ and $y_{i} \mid y_{i+1}$ for all $i$. Dividing all $y_{i}$ 's by an appropriate number, we may assume that $x_{i}=y_{i}$ for some $i$. Since $x_{d}<2$, we must have $y_{j}=x_{i}$ for all $j \leq i$ and of course $y_{j} \geq 2 x_{i}$ for all $j>i$. Thus,

$$
\frac{y_{1} \cdots y_{d}}{x_{1} \cdots x_{d}} \geq 2^{\frac{i-1}{d}} \cdot 2^{\frac{i-2}{d}} \cdots 1 \cdot 2^{\frac{d-1}{d}} \cdot 2^{\frac{d-2}{d}} \cdots 2^{\frac{i}{d}}=2^{\frac{d-1}{2}} .
$$


Since $y_{1}, \ldots, y_{d}$ is an arbitrary sequence with the above properties, we finally show that $c_{d}=2^{(d-1) / 2}$.

It is a more difficult task to compute $C_{d}$ exactly; we will provide an upper bound, however.

Proof of Proposition 1.1 The averaging process is slightly different than before; for each integer $a$ with $x_{1} \leq a<2 x_{1}$, we construct a sequence $y_{1}^{a}, \ldots, y_{d}^{a}$ satisfying $y_{1}^{a}=a$ and

$$
y_{i}^{a}=2^{b_{a i}} a, \quad \text { where } b_{a i}=-\left[\log _{2} a-\log _{2} x_{i}\right] .
$$

As before,

$$
\log _{2} \frac{y_{1}^{a} \cdots y_{d}^{a}}{x_{1} \cdots x_{d}}=\sum_{i=1}^{d}\left\{\log _{2} a-\log _{2} x_{i}\right\} .
$$

Summing over all $a$, we obtain

$$
\sum_{a=x_{1}}^{2 x_{1}-1} \log _{2} \frac{y_{1}^{a} \cdots y_{d}^{a}}{x_{1} \cdots x_{d}}=\sum_{a=x_{1}}^{2 x_{1}-1} \sum_{i=1}^{d}\left\{\log _{2} a-\log _{2} x_{i}\right\}
$$

For $i=1$, we obtain

$$
\sum_{a=x_{1}}^{2 x_{1}-1}\left\{\log _{2} \frac{a}{x_{1}}\right\}<x_{1} \int_{1}^{2} \log _{2} x d x
$$

Now let $i>1$. The following equality holds:

$$
\left\{\log _{2} a-\log _{2} x_{i}\right\}=\left\{\log _{2} \frac{a}{x_{1}}\right\}-\left\{\log _{2} \frac{x_{i}}{x_{1}}\right\}+\varepsilon,
$$

where $\varepsilon=0$ or 1 , depending on whether $\left\{\log _{2} \frac{x_{i}}{x_{1}}\right\} \leq\left\{\log _{2} \frac{a}{x_{1}}\right\}$ or $\left\{\log _{2} \frac{x_{i}}{x_{1}}\right\}>$ $\left\{\log _{2} \frac{a}{x_{1}}\right\}$. If $\left\{\log _{2} \frac{x_{i}}{x_{1}}\right\}=0$, then we get the same result as in the case $i=1$. Otherwise, let $l$ be the unique integer satisfying

$$
\log _{2} \frac{x_{1}+l-1}{x_{1}}<\left\{\log _{2} \frac{x_{i}}{x_{1}}\right\} \leq \log _{2} \frac{x_{1}+l}{x_{1}} .
$$

Of course, $1 \leq l \leq x_{1}$. Thus, we obtain

$$
\begin{aligned}
\sum_{a=x_{1}}^{2 x_{1}-1}\left\{\log _{2} \frac{a}{x_{i}}\right\} & =\sum_{a=x_{1}}^{2 x_{1}-1} \log _{2} \frac{a}{x_{1}}-x_{1}\left\{\log _{2} \frac{x_{i}}{x_{1}}\right\}+l \\
& <\sum_{a=x_{1}}^{2 x_{1}-1} \log _{2} \frac{a}{x_{1}}-x_{1} \log _{2} \frac{x_{1}+l-1}{x_{1}}+l
\end{aligned}
$$




$$
\begin{aligned}
& =\sum_{a=x_{1}}^{2 x_{1}-1} \log _{2} \frac{a}{x_{1}}-\log _{2}\left(1+\frac{l-1}{x_{1}}\right)^{x_{1}}+l \\
& <\sum_{a=x_{1}}^{2 x_{1}-1} \log _{2} \frac{a}{x_{1}}-\log _{2} 2^{l-1}+l \\
& =\sum_{a=x_{1}}^{2 x_{1}-1} \log _{2} \frac{a}{x_{1}}+1 \\
& =\sum_{a=x_{1}+1}^{2 x_{1}} \log _{2} \frac{a}{x_{1}} .
\end{aligned}
$$

The latter is an upper Riemann sum, multiplied by $x_{1}$, for the function $f(x)=\log _{2} x$ for the partition

$$
1=\frac{x_{1}}{x_{1}}<\frac{x_{1}+1}{x_{1}}<\cdots<\frac{2 x_{1}-1}{x_{1}}<\frac{2 x_{1}}{x_{1}}=2 .
$$

It is a simple task to prove that

$$
\frac{1}{x_{1}} \sum_{a=x_{1}+1}^{2 x_{1}} \log _{2} \frac{a}{x_{1}}
$$

is decreasing in $x_{1}$ and converging, of course, to $\int_{1}^{2} \log _{2} x d x$. Without loss of generality, we may assume that $x_{1} \geq 2$; otherwise, we disregard all terms equal to 1 , because we can set $y_{i}=x_{i}=1$, and we consider the first term of the sequence $x_{1}, \ldots, x_{d}$ which is greater than 1 . So, the maximal value of the Riemann sum is

$$
\frac{1}{2}\left(\log _{2} \frac{3}{2}+\log _{2} \frac{4}{2}\right)=\log _{2} \sqrt{3},
$$

and hence

$$
\sum_{a=x_{1}}^{2 x_{1}-1}\left\{\log _{2} \frac{a}{x_{i}}\right\}<x_{1} \log _{2} \sqrt{3}
$$

Thus,

$$
\sum_{a=x_{1}}^{2 x_{1}-1} \sum_{i=1}^{d}\left\{\log _{2} a-\log _{2} x_{i}\right\}<x_{1}\left(\int_{1}^{2} \log _{2} x d x+(d-1) \log _{2} \sqrt{3}\right)
$$

therefore, there is a number $a$ for which the following inequality holds:

$$
\sum_{i=1}^{d}\left\{\log _{2} a-\log _{2} x_{i}\right\}<2-\frac{1}{\ln 2}+(d-1) \log _{2} \sqrt{3},
$$


so finally

$$
\frac{y_{1}^{a} \cdots y_{d}^{a}}{x_{1} \cdots x_{d}}<\frac{4}{e} \cdot 3^{(d-1) / 2},
$$

as desired.

As for the other inequality, we will base our arguments on the example at the end of Lemma 3.1, which shows $c_{d} \geq 2^{(d-1) / 2}$. We will actually prove that for all $\delta>0$, the following inequality holds:

$$
C_{d}>(1-\delta)^{\frac{d(d-1)}{2}} \cdot 2^{\frac{d-1}{2}} .
$$

Let $\delta>0$ be arbitrary, and let $M$ be a positive integer such that

$$
M>\frac{1}{\delta \sqrt[d]{2}} .
$$

We define then $x_{1}=M, x_{i+1}=\left[x_{i} \sqrt[d]{2}\right]$ for $1 \leq i \leq d-1$. Let $y_{1}, \ldots, y_{d}$ be a sequence of positive integers satisfying $x_{i} \leq y_{i}$ and $y_{i} \mid y_{i+1}$ for all $i$ and such that the product $y_{1} y_{2} \cdots y_{d}$ is minimal. Since $x_{d}<2 x_{1}$, we deduce that $y_{d}=y_{1}$ or $2 y_{1}$. If $y_{1}=y_{d}$, then by the minimality assumption, $y_{i}=x_{d}$ for all $i$. Otherwise, let $i$ be the maximal index such that $y_{i}=x_{i}$ (i.e., $y_{1}=y_{2}=\cdots=y_{i}, 2 y_{i}=y_{i+1}=\cdots=y_{d}$ ). Then again, by minimality we have that $y_{i}=x_{i}$. So, the sequence $y_{1}, \ldots, y_{d}$ has the form

$$
\underbrace{x_{i}, \ldots, x_{i}}_{i \text { terms }}, \underbrace{2 x_{i}, \ldots, 2 x_{i}}_{d-i \text { terms }}
$$

for some index $i$. We will prove that we actually have $i=d$. Indeed, from the definition of the sequence $\left\{x_{i}\right\}_{i=1}^{d}$ we have that

$$
\frac{x_{i} \cdot \sqrt[d]{2}-1}{x_{i}}<\frac{x_{i+1}}{x_{i}}<\sqrt[d]{2},
$$

which implies

$$
\sqrt[d]{2}-\frac{1}{M}<\frac{x_{i+1}}{x_{i}}<\sqrt[d]{2}
$$

and since $M>1 /(\delta \sqrt[d]{2})$, we get

$$
(1-\delta) \sqrt[d]{2}<\frac{x_{i+1}}{x_{i}}<\sqrt[d]{2}
$$

thus, for $j>i$,

$$
(1-\delta)^{j-i} \cdot 2^{\frac{j-i}{d}}<\frac{x_{j}}{x_{i}}<2^{\frac{j-i}{d}} .
$$

For $j=d$, the right-hand side becomes

$$
\left(\frac{x_{d}}{x_{i}}\right)^{d}<2^{d-i}
$$


or

$$
x_{d}^{d}<2^{d-i} x_{i}^{d}=\underbrace{x_{i} \cdots x_{i}}_{i \text { terms }} \cdot \underbrace{2 x_{i} \cdots 2 x_{i}}_{d-i \text { terms }} .
$$

So, we proved that $y_{i}=x_{d}$ for all $i$. Using the left-hand side inequalities of (3), for $j=d$, we obtain

$$
\prod_{i=1}^{d-1} \frac{x_{d}}{x_{i}}>(1-\delta)^{\frac{d(d-1)}{2}} \cdot 2^{\frac{d-1}{2}}
$$

hence,

$$
C_{d}>(1-\delta)^{\frac{d(d-1)}{2}} \cdot 2^{\frac{d-1}{2}}
$$

for all $\delta>0$, and thus

$$
C_{d} \geq 2^{\frac{d-1}{2}}
$$

completing the proof.

Proof of Theorem 1.4 We can make a further improvement; let $k$ be the smallest index such that $\lambda_{k}>1$. If $k=1$, then $G(K)=1$, and the conjecture is verified. If $k>1$, then we have a reduction to fewer dimensions, namely $k-1$, because $K \cap \Lambda$ has at most $k-1$ linearly independent vectors, by the definition of the successive minima. So, if we intersect $K$ and $\Lambda$ with the linear hull of these vectors, we get a $(k-1)$-dimensional convex body $K^{\prime}$ and a $(k-1)$-dimensional lattice $\Lambda^{\prime}$ such that $\lambda_{i}\left(K^{\prime}, \Lambda^{\prime}\right) \leq 1$ for all $i$. Furthermore, $G(K, \Lambda)=G\left(K^{\prime}, \Lambda^{\prime}\right)$. This shows that we can reduce to the case where all successive minima are less than or equal to 1 . In this case, all $q_{i}$ are at least equal to 3 .

Combining this observation with the proof of Proposition 1.1, we can see that we can take $x_{1} \geq 3$ for the purposes of our geometric problem. Therefore, the maximal value for the upper Riemann sum

$$
\frac{1}{x_{1}} \sum_{a=x_{1}+1}^{2 x_{1}} \log _{2} \frac{a}{x_{1}}
$$

is obtained for $x_{1}=3$, which is

$$
\frac{1}{3}\left(\log _{2} \frac{4}{3}+\log _{2} \frac{5}{3}+\log _{2} \frac{6}{3}\right)=\log _{2} \sqrt[3]{\frac{40}{9}}
$$

Therefore, the corresponding constant, under the restriction $x_{1} \geq 3$, is less than or equal to

$$
\frac{4}{e}\left(\frac{40}{9}\right)^{\frac{d-1}{3}} \approx 1.47152 \cdot 1.64414^{d-1},
$$

concluding the proof. 
We continue with proving the following property of an optimal solution:

Lemma 3.2 Let $x_{1} \leq x_{2} \leq \cdots \leq x_{d}$ be a finite sequence of $d$ positive integers. Let $y_{1}, \ldots, y_{d}$ be the "optimal" sequence of integers with respect to $x_{1}, \ldots, x_{d}$, i.e., satisfying $x_{i} \leq y_{i}, y_{i} \mid y_{i+1}$ for all $i$, such that the product of all $y_{i}$ is minimal. Then $y_{d}<2 x_{d}$.

Proof Assume otherwise, and let $i$ be the smallest index such that $y_{i} \geq 2 x_{i}$. If $i=1$, then

$$
\frac{y_{1} \cdots y_{d}}{x_{1} \cdots x_{d}} \geq 2^{d}
$$

which contradicts the minimality of the product, as it can be seen from Henk's proof. So $i>1$, and $y_{i-1}<2 x_{i-1}$. Define $y_{i}^{\prime}$ to be the least multiple of $y_{i-1}$ exceeding $x_{i}$, and inductively for $i<j \leq d$, define $y_{j}^{\prime}$ to be the least multiple of $y_{j-1}^{\prime}$ exceeding $x_{j}$. Since $y_{i-1}<2 x_{i-1}$, it is not hard to see that $y_{i}^{\prime}<2 x_{i} \leq y_{i}$ and inductively that $y_{j}^{\prime}<$ $2 x_{j} \leq y_{j}$; thus,

$$
y_{1} \cdots y_{i-1} \cdot y_{i}^{\prime} \cdots y_{d}^{\prime}<y_{1} \cdots y_{d},
$$

contradicting the minimality of the product $y_{1} \cdots y_{d}$. Thus $y_{d}<2 x_{d}$, as desired.

At this point, we should mention some values of $C_{d}$, for small $d$ :

\begin{tabular}{llll}
\hline$d$ & 2 & 3 & 4 \\
\hline$C_{d}$ & $\sqrt{2}$ & $250 / 117$ & $16384 / 5187$ \\
\hline
\end{tabular}

The proof for $C_{2}=\sqrt{2}$ is easy; $C_{3}$ and $C_{4}$ need case-by-case examination, which can finally be reduced to finitely many $d$-tuples $x_{1}, \ldots, x_{d}$ for $d=3$ or 4 . Those were checked by a simple computer program, written in GWBASIC 3.23, verifying the values above. The optimal solutions were obtained from $x_{1}=4, x_{2}=9, x_{3}=13$ and $y_{1}=5, y_{2}=10, y_{3}=20$ for $d=3$, and from $x_{1}=3, x_{2}=7, x_{3}=13, x_{4}=19$ and $y_{1}=4, y_{2}=8, y_{3}=16, y_{4}=32$ for $d=4$.

\section{A Minor Improvement}

By reexamining Henk's proof, we can see that we need not require $n_{2} \mid n_{1}$; indeed, if this index $k$ is either equal to 1 or $\geq 3$, then the proof is the same. We need, however, that $n_{3} \mid n_{1}$. If $k=2$, we will use the same convexity argument that was used to verify the conjecture for $d=2$; that is, we may take $e_{1} \in \lambda_{1} K$ and $e_{2} \in \lambda_{2} K \backslash \operatorname{int}\left(\lambda_{2} K\right)$. Then, we can easily deduce that

$$
\frac{n_{1}}{\left|n_{1}\right|} e_{1}+\frac{n_{2}}{\left|n_{2}\right|} e_{2} \in \operatorname{int}\left(\operatorname{conv}\left\{0, e_{1}, e_{2}, \lambda_{2} g\right\}\right)
$$


if $n_{1} \neq 0$ or

$$
\frac{n_{2}}{\left|n_{2}\right|} e_{2} \in \operatorname{int}\left(\operatorname{conv}\left\{0, e_{1}, e_{2}, \lambda_{2} g\right\}\right)
$$

otherwise, contradicting the definition of $\lambda_{2}$. For a proof of these statements, see Theorem 2.2 in [3].

Hence, we may drop the requirement that $y_{d-1}$ divides $y_{d}$ in the arithmetic problem (replacing it by $y_{d-2} \mid y_{d}$ ):

Definition 4.1 Let $C_{d}^{\prime}$ denote the least positive constant such that for any nondecreasing sequence of integers $x_{1}, x_{2}, \ldots, x_{d}$, there exists a sequence of integers $y_{1}, y_{2}, \ldots, y_{d}$ satisfying:

(a) $x_{i} \leq y_{i}$ for all $i, 1 \leq i \leq d$.

(b) $y_{i}$ divides $y_{i+1}$ for all $i, 1 \leq i \leq d-2$, and $y_{d-2}$ divides $y_{d}$.

(c) $\frac{y_{1} y_{2} \cdots y_{d}}{x_{1} x_{2} \cdots x_{d}} \leq C_{d}^{\prime}$.

This constant obviously satisfies $c_{d} \leq C_{d}^{\prime} \leq C_{d}$ for $d \geq 3$. The technique in the proof of Proposition 1.1 does not provide a better bound in the general case; however, $C_{d}^{\prime}$ is more easily computable for small values of $d$.

We will also need the following lemma:

Lemma 4.1 Let $x_{1} \leq x_{2} \leq \cdots \leq x_{d} \leq 2 x_{1}$ be a finite sequence of $d$ positive integers. There is always an increasing sequence of $d$ positive integers $y_{1}, \ldots, y_{d}$ satisfying $y_{i} \mid y_{i+1}, x_{i} \leq y_{i}$ for all $i$ and

$$
\frac{y_{1} \cdots y_{d}}{x_{1} \cdots x_{d}} \leq 2^{\frac{d-1}{2}}
$$

Proof For each $i$, we construct the sequence $y_{1}^{i}, \ldots, y_{d}^{i}$ satisfying

$$
y_{1}^{i}=\cdots=y_{i}^{i}=x_{i}, \quad y_{i+1}^{i}=\cdots=y_{d}^{i}=2 x_{i} .
$$

Obviously, each of these $d$ sequences satisfies $y_{j}^{i} \mid y_{j+1}^{i}$ and $x_{j} \leq y_{j}^{i}$. Furthermore,

$$
\prod_{i=1}^{d} \frac{y_{1}^{i} \cdots y_{d}^{i}}{x_{1} \cdots x_{d}}=2^{\frac{d(d-1)}{2}},
$$

and thus there is at least one $i$ such that

$$
\frac{y_{1}^{i} \cdots y_{d}^{i}}{x_{1} \cdots x_{d}} \leq 2^{\frac{d-1}{2}} .
$$

4.1 The Case $d=3$

Theorem 4.1 $C_{3}^{\prime}=2$. 
Proof In each of the following cases for $x_{1}, x_{2}, x_{3}$, we will construct three integers $y_{1}, y_{2}, y_{3}$ satisfying $x_{i} \leq y_{i}, y_{1}\left|y_{2}, y_{1}\right| y_{3}$, and

$$
\frac{y_{1} y_{2} y_{3}}{x_{1} x_{2} x_{3}} \leq 2 \text {. }
$$

Case $1 x_{2} \geq \frac{3}{\sqrt{2}} \cdot x_{1}$ : We put $y_{1}=x_{1}$, and let $y_{2}, y_{3}$ be the least multiples of $x_{1}$ exceeding $x_{2}, x_{3}$, respectively. By hypothesis, we will have $y_{i} / x_{i} \leq \sqrt{2}$ for $i=2,3$, and thus

$$
\frac{y_{1} y_{2} y_{3}}{x_{1} x_{2} x_{3}} \leq 2
$$

Case $2 x_{1}<x_{2}<\frac{3}{\sqrt{2}} \cdot x_{1}$ : The existence of an integer strictly between $2 x_{1}$ and $\frac{3}{\sqrt{2}} \cdot x_{1}$ implies $x_{1} \geq 9$. If $x_{3} \geq \frac{9}{4} \cdot x_{1}$, then we put $y_{1}=x_{1}$, and let $y_{2}, y_{3}$ be the least multiples of $x_{1}$ exceeding $x_{2}, x_{3}$, respectively. By hypothesis, $y_{2} / x_{2}<3 / 2$ and $y_{3} / x_{3} \leq 4 / 3$, and thus

$$
\frac{y_{1} y_{2} y_{3}}{x_{1} x_{2} x_{3}} \leq 2
$$

If $x_{3}<\frac{9}{4} \cdot x_{1}$, then if $x_{3}$ is even, we put $y_{1}=x_{3} / 2, y_{2}=y_{3}=x_{3}$. We will have

$$
\frac{y_{1} y_{2} y_{3}}{x_{1} x_{2} x_{3}}<\left(\frac{x_{3}}{2 x_{1}}\right)^{2}<\left(\frac{9}{8}\right)^{2}<1.27 \text {. }
$$

If $x_{3}$ is odd, we put $y_{1}=\left(x_{3}+1\right) / 2, y_{2}=y_{3}=x_{3}+1$. Then

$$
\begin{aligned}
\frac{y_{1} y_{2} y_{3}}{x_{1} x_{2} x_{3}} & <\left(\frac{\frac{9}{4} \cdot x_{1}+1}{2 x_{1}}\right)^{2} \cdot \frac{x_{3}+1}{x_{3}}=\left(\frac{9}{8}+\frac{1}{2 x_{1}}\right) \cdot\left(1+\frac{1}{x_{3}}\right) \\
& \leq\left(\frac{9}{8}+\frac{1}{18}\right) \cdot\left(1+\frac{1}{2 x_{1}+1}\right) \leq \frac{85}{72} \cdot \frac{20}{19}<2 .
\end{aligned}
$$

Case $3 \sqrt{2} \cdot x_{1} \leq x_{2} \leq 2 x_{1}$ : If either $x_{3} \leq 2 x_{1}$ or $x_{3} \geq \frac{3}{\sqrt{2}} \cdot x_{1}$, then we put $y_{1}=x_{1}$, and let $y_{2}, y_{3}$ be the least multiples of $x_{1}$ exceeding $x_{2}, x_{3}$, respectively. By hypothesis, we will have $y_{i} / x_{i} \leq \sqrt{2}$ for $i=2,3$, and thus

$$
\frac{y_{1} y_{2} y_{3}}{x_{1} x_{2} x_{3}} \leq 2
$$

Suppose now that $2 x_{1}<x_{3}<\frac{3}{\sqrt{2}} \cdot x_{1}$. Then, $x_{1} \geq 9$, and we put $y_{3}=x_{3}$ if $x_{3}$ is even and $y_{3}=x_{3}+1$ otherwise. Then, we put $y_{1}=y_{3} / 2$ and $y_{2}=y_{3}$. It is not hard to verify that $\left(y_{1} y_{3}\right) /\left(x_{1} x_{3}\right)<1.18$ and

$$
\frac{y_{2}}{x_{2}}<\frac{\frac{3}{\sqrt{2}} \cdot x_{1}+1}{\sqrt{2} \cdot x_{1}}=\frac{3}{2}+\frac{1}{\sqrt{2} \cdot x_{1}}<\frac{3}{2}+\frac{1}{12}=\frac{19}{12} .
$$


Thus,

$$
\frac{y_{1} y_{2} y_{3}}{x_{1} x_{2} x_{3}}<\frac{19}{12} \cdot 1.18<2 .
$$

Case $4 x_{2}<\sqrt{2} \cdot x_{1}$ : If $x_{3}<\sqrt{2} \cdot x_{2}$, then $x_{3}<2 x_{1}$, and we apply Lemma 4.1. If either $\sqrt{2} \cdot x_{2} \leq x_{3} \leq 2 x_{2}$ or $x_{3} \geq \frac{3}{\sqrt{2}} \cdot x_{2}$, we put $y_{1}=y_{2}=x_{2}$, and let $y_{3}$ be the least multiple of $x_{2}$ exceeding $x_{3}$. Then, $y_{1} / x_{1}<\sqrt{2}$ and $y_{3} / x_{3} \leq \sqrt{2}$, and thus

$$
\frac{y_{1} y_{2} y_{3}}{x_{1} x_{2} x_{3}}<2
$$

It only remains to check the case $2 x_{2}<x_{3}<\frac{3}{\sqrt{2}} \cdot x_{2}$. We will have $x_{2} \geq 9$ and $x_{1} \geq 7$. As in case 3 above, we can find integers $y_{2}, y_{3}$ such that $x_{i} \leq y_{i}, y_{2} \mid y_{3}$, and

$$
\frac{y_{2} y_{3}}{x_{2} x_{3}}<1.18
$$

In particular, $y_{2} / x_{2}<1.18$. Now, we put $y_{1}=y_{2}$. We will have $y_{1} / x_{1}=\left(y_{2} / x_{2}\right)$. $\left(x_{2} / x_{1}\right)<1.18 \cdot \sqrt{2}$, and thus

$$
\frac{y_{1} y_{2} y_{3}}{x_{1} x_{2} x_{3}}<\sqrt{2} \cdot 1.18^{2}<2
$$

completing the proof.

Theorem 4.1 clearly implies the first part of Proposition $1.2(d=3)$.

\subsection{The Case $d=4$}

Even though $C_{4}^{\prime}$ is more easily computable than $C_{4}$, computer tests could not be avoided. The optimal quadruplets were $x_{1}=6, x_{2}=15, x_{3}=19, x_{4}=25$ and $y_{1}=7$, $y_{2}=y_{3}=21, y_{4}=42$. They yield

$$
\frac{y_{1} y_{2} y_{3} y_{4}}{x_{1} x_{2} x_{3} x_{4}}=\frac{7203}{2375} \approx 3.032842105263157894736842105263157894736 \ldots
$$

This proves the second part of Proposition $1.2(d=4)$.

Open Access This article is distributed under the terms of the Creative Commons Attribution Noncommercial License which permits any noncommercial use, distribution, and reproduction in any medium, provided the original author(s) and source are credited.

\section{References}

1. Betke, U., Henk, M., Wills, J.M.: Successive-minima-type inequalities. Discrete Comput. Geom. 9(2), 165-175 (1993)

2. Gruber, P.M., Lekkerkerker, C.G.: Geometry of Numbers, 2nd edn. North-Holland, Amsterdam (1987)

3. Henk, M.: Successive minima and lattice points. Rend. Circ. Mat. Palermo Ser. II Suppl. 70, 377-384 (2002)

4. Rogers, C.A.: The product of the minima and the determinant of a set. Proc. K. Ned. Akad. Wet. 52, 256-263 (1949) 